DOI: $10.17234 / S R A Z .65 .40$

\title{
"Le femmine di panza si sentono attapanate?": Reflexiones traductológicas sobre la interpretación castellana del lenguaje literario siciliano
}

\author{
José García Fernández \\ Universidad de Oviedo (España) \\ garciafernandezjose@uniovi.es
}

\begin{abstract}
El lenguaje literario de Giuseppina Torregrossa destila la esencia definitoria de su tierra natal: Sicilia. La "sicilianidad" de sus textos evoca la opresora condición de la mujer isleña y pone de manifiesto la "mafiosidad" de un territorio que en ocasiones vive instalado en el secretismo del silencio. Acercándonos a estas singularidades culturales, este artículo ahonda en el ánima femenina de la isla, no siempre fácil de dar a conocer a los lectores hispanos. De carácter práctico y traductológico, esta publicación subraya las dificultades que conlleva la traducción al castellano de dos sintagmas sicilianos: attapanata 'enchironada' y femmina di panza 'mujer reservada', dos expresiones de marcado tinte etnográfico cuyo análisis demostrará cuán representativas son de la milenaria cultura siciliana, permitiendo a su vez responder a la pregunta que da título a este trabajo: Le femmine di panza si sentono attapanate? '¿Las mujeres reservadas se sienten enchironadas?'
\end{abstract}

Palabras clave: práctica traductológica, dificultades de traducción, cultura siciliana, traducción siciliano-español, Giuseppina Torregrossa.

\section{Introducción, metodología y objetivos}

El lenguaje literario siciliano refleja lingüísticamente la situación social de las mujeres de la isla. Obligadas a guardar silencio, muchas sicilianas se sintieron atrapadas en sí mismas por culpa de hombres (esposos, padres, hijos, etc.) que acabarían por involucrarlas en sus prácticas criminales (Faccioli 1983: 110-133; Dino 2000: 74-91; Fiandaca 2003; Pasculli 2009: 80-97). Consciente de esta situación, la escritora palermitana Giuseppina Torregrossa (1956-) plasma en su producción literaria las características culturales de un territorio heterogéneo que presenta al lector mediante el uso de diversas expresiones dialectales sicilianas. En concreto, este artículo se detiene en el análisis traductológico de los sintagmas femmina di panza y attapanata, dos expresiones lingüísticas empleadas por Torregrossa tanto en L'assaggiatrice (2010a) como en Il conto delle minne (2010b). ${ }^{1}$ En estas dos novelas

1 La investigación en la que se basa este artículo se ha desarrollado gracias al proyecto

“Men for Women. Voces Masculinas en la Querella de las Mujeres" (PID2019-104004GB- 
la autora logra definir la "sicilianidad" femenina de su tierra natal, enlazándola incluso algunas veces con la "mafiosidad" isleña (Dino/Meli 1997; Dino 1998: 477-512; Strazzeri 2016: 167-174). Esta particularidad estilística ha sido tomada en consideración por parte del traductor de ambas obras, coincidente, por otra parte, con quien suscribe este artículo. ${ }^{2}$

Sabedores de la importancia de estar atentos al Weltanschauung siciliano (esto es, a la visión del mundo siciliana), las elecciones traductológicas se han llevado a cabo de acuerdo con la idiosincrasia del marco narrativo del relato: Sicilia. Sin embargo, a nivel metodológico, aun siguiendo el principio de fidelidad al texto original, se ha considerado igualmente esencial la alternancia de la traducción comunicativa y semántica (Newmark 1977: 163-180, 2010). En consecuencia, se ha procurado no alterar demasiado el mensaje contenido en los libros de Torregrossa, reflexionando para ello sobre la interpretación más apropiada de los dos sintagmas arriba mencionados.

Sacando a la luz la complejidad de estos aspectos sociales y lingüísticos a través del estudio traductológico de varios ejemplos extraídos de dos volúmenes de Torregrossa (2010a, 2010b), este artículo persigue: 1) determinar los obstáculos derivados de la traducción al español del texto original siciliano; 2) advertir algunas de las singularidades etnográficas y sociales de la actual Sicilia; y 3) examinar el estilo lingüístico de la escritora con el fin de ver hasta qué punto este se ha respetado en la traducción castellana.

\section{2. Èssiri attapanata: ¿sentirse enchironada?}

De acuerdo con lo explicitado en la sección anterior, el traductor se ha atenido al principio de fidelidad al texto y a la escritora, estimando fundamental alternar la traducción comunicativa y semántica. Se ha intentado, por consiguiente, no transmutar en exceso el mensaje contenido en las tramas de Torregrossa, optándose solamente por modificar algunas estructuras en aquellos fragmentos que pudieran resultar complicados de entender por parte de un lector hispano (e incluso italiano). ${ }^{3}$ Esta ardua tarea se constata en los siguientes casos: ${ }^{4}$

(1) a. Texto original: Io mi sento attapanata. Cosa vuol dire? Non lo so con precisione, è una parola che sentivo a casa da bambina. Mia madre, quando

I00). Dicho proyecto está financiado por el Ministerio de Ciencia e Innovación del Gobierno de España.

2 Las traducciones del autor son inéditas. No obstante, cabe señalar que Il conto delle minne ya ha sido traducido al castellano por Teresa Clavel en 2011 bajo el título Un dulce par de senos.

3 En cualquier caso, también se ha tenido en cuenta en el proceso de traducción que "un factor indispensable es que exista empatía entre el traductor y el TLO [Texto de la Lengua Original] y su autor, esto es, que el traductor ha de sentir el texto en toda su complejidad, tanto lingüística como extralingüística, y participar tanto afectiva como emotivamente con el autor y su obra" (González de Sande 2009: 467).

4 Todas las traducciones al castellano de este artículo son propias. 
non ne poteva più di lavorare come una bestia, diceva che si sentiva attapanata (Torregrossa 2010a: 16).

b. Texto en castellano: Me siento enchironada. ¿Qué quiere decir? No lo sé con precisión, es una palabra que escuchaba en casa de pequeña. Mi madre, cuando estaba harta de trabajar como un burro, decía que se sentía enchironada.

(2) a. Texto original: Mi sono fatta l'idea che [essere attapanata] è come essere in trappola, anzi in una palude, i piedi affondati nelle sabbie mobili, il corpo per intero nel fango, la testa fuori per respirare. Quando ogni piccolo movimento produce uno sprofondare ulteriore, ecco allora sei attapanata (Torregrossa 2010a: 16-17).

b. Texto en castellano: Me he hecho a la idea de que [estar enchironada] es como estar en una trampa, más bien en un pantano, con los pies hundidos en arenas movedizas, el cuerpo por completo en el fango, la cabeza fuera para respirar. Cuando cada pequeño movimiento te hunde aún más, es entonces cuando estás enchironada.

(3) a. Texto original: [Gaetano] mi diceva: "Ancilù e che cosa ti manca? Che deve dire la gente che l'ingegnere soldi a casa non ne porta?" E così mi tappava la bocca e mi legava le mani. Incaprettata ero, anzi attapanata! (Torregrossa 2010a: 24).

b. Texto en castellano: [Gaetano] me decía: “Ancilù, ¿qué te falta? ¿Qué quieres que diga la gente, que el ingeniero no trae dinero a casa?" $\mathrm{Y}$ así me cerraba la boca y me ataba las manos. Estaba sometida o más bien jenchironada!

Basados en el adjetivo attapanata, estos ejemplos dan muestra de la dificultad que en ocasiones acarrea la traducción al castellano de ciertos términos, no ya italianos, sino propios del siciliano. En estos fragmentos la voz attapanata está relacionada con un estado anímico de desconcierto, turbación y falta de libertad, 5 un simbolismo que al mismo tiempo saca a la luz la sumisión y el despotismo sufridos por muchas sicilianas que no tuvieron más opción que resignarse a sobrellevar una vida social y patriarcalmente impuesta.

Prestando atención a este contexto de subyugación al que Torregrossa alude en L'assaggiatrice, la empatía del traductor (Bouzalmate 1995: 93-97) ha sido la que ha motivado la elección del término jergal español enchironar, un vocablo que, además de reproducir el coloquialismo dialectal recogido por la autora en el texto original, también evidencia la paulatina pérdida de estabilidad mental de la que Anciluzza, protagonista de L'assaggiatrice, fue víctima. De hecho, la inclusión de esta palabra

5 El término attapanata procede de attipanári, forma sícula que remite al verbo siciliano tipári, cuyo étimo latino sería STĪPĀRE y cuyo significado en italiano es 'stipare, ammassare, calcare' (Varvaro 2014: 1079). En relación con calcare, la versión telemática del Vocabolario Treccani matiza que se puede utilizar a nivel literario de forma figurativa con la significación de 'oprimir, humillar' o sinónimos equivalentes. Dicho contenido ha permitido al traductor interpretar el adjetivo empleado por Torregrossa, un adjetivo deverbal que pone de relieve el desgaste emocional de Anciluzza, personaje central de L'assaggiatrice. 
en la trama no es casual, sino que anuncia el comienzo de un proceso de cambio en el que Anciluzza, tras la angustia derivada del abandono de su esposo, acabaría por superar su inicial aturdimiento y por convertirse en una mujer completamente renovada. Así pues, no es de extrañar que esta voz aparezca al principio de la obra, momento en el que Torregrossa se sirve de ella para intensificar el drama de la acción y el grado de desconcierto en el que se hallaba la protagonista.

\section{Fimmina di panza: ¿mujer reservada?}

En el apartado precedente se ha puesto de manifiesto la importancia de ser fieles al texto original, así como la necesidad de alternar las traducciones semántica y comunicativa para dotar de mayor calidad a la traducción final. No obstante, se ha considerado igualmente oportuno llevar a cabo la traducción de manera retrospectiva, es decir, intentando respetar a la escritora y su modo de pensar, y procurando dejar intacta la esencia cultural siciliana que brota del lenguaje de Torregrossa. Destáquense los siguientes ejemplos:

(4) a. Texto original: Vito è uno che zitto non ci può stare [...] In paese lo chiamiamo il cucchiaio di tutte le pignate, perché fa domande in giro, chiede a tutti informazioni e poi riferisce a sua moglie, vera femmina di panza, che mai l'ho sentita parlare male di qualcuno, ma neanche bene, proprio non parla (Torregrossa 2010a: 95).

b. Texto en castellano: Vito es alguien que no puede estar callado [...] En el pueblo lo llamamos la cuchara de todas las cacerolas, porque hace preguntas por ahí, pide información a todo el mundo y luego informa a su mujer, auténtica mujer reservada, a la que nunca he escuchado hablar mal de nadie, pero tampoco bien, simplemente no habla.

(5) a. Texto original: La nonna Margherita, che in quei primi anni palermitani aveva goduto di una vita discretamente agiata, [...] sprofondò dalle luci della città al buio della provincia agricola. Ma dei tormenti e delle difficoltà che sicuramente l'accompagnarono in questo improvviso trasloco sappiamo poco o nulla: di sé non ha mai parlato, vera femmina di panza (Torregrossa 2010b: 112).

b. Texto en castellano: La abuela Margherita, que en sus primeros años en Palermo había disfrutado de una posición discretamente acomodada, [...] pasó de las luces de la ciudad a la oscuridad de la provincia agrícola. Pero poco sabemos de los tormentos y de las dificultades que seguramente le acompañaron en esta repentina mudanza: de sí misma nunca ha hablado, auténtica mujer reservada.

En estos ejemplos, en búsqueda de un equivalente idiomático en castellano, el traductor se ha visto obligado a hacer una traducción parcial del significado

6 Aunque originalmente referida a un varón, el Vocabolario siciliano explica cómo esta expresión alude a la "persona che sa mantenere un segreto o tutto ciò che deve restare segreto, soprattutto in ambiente mafioso" (Tropea 1990: 553-554). No sorprende, por ende, que Torregrossa haya empleado esta misma fórmula lingüística para referirse a las mujeres, tal y como han hecho otras literatas sicilianas de renombre como 
primigenio. En lugar de explicar la expresión femmina di panza, ${ }^{6}$ perteneciente al lenguaje de la mafia -y con la que, además, se hace mención a una mujer que sabe estar callada y que se atiene a lo que en italiano se conoce como omertà 'ley del silencio' (Di Bella 2011)-, el traductor ha realizado una interpretación del texto original y ha preferido alterar el mensaje de Torregrossa al utilizar la construcción léxica mujer reservada. El traductor se ha servido así de un sintagma en el que quedaría recogida grosso modo la acepción a la que Torregrossa alude en sus obras, si bien, tal y como señala G. Mounin, tratando de "estraniare il lettore [...] dal suo mondo, decidendo di fargli leggere il testo senza permettergli di dimenticare un solo istante che si trova di fronte a [...] una civiltà diversa" (Mounin 1999: 140). En otras palabras, de conformidad con los planteamientos de M. González de Sande, el propósito de la traducción ha sido:

Conservar la forma, estilo y cultura del TLO [Texto de la Lengua Original], de manera que el lector del TLT [Texto de la Lengua Terminal] pueda sentirse ante una obra perteneciente a otro mundo, otro país, otra cultura, acercándose, a través de la lectura del texto, a este, y, por tanto, recibiendo (siempre dentro de las limitaciones que implica la no pertenencia a dicho mundo) el mismo efecto que produjo en el lector del texto original (González de Sande 2009: 474).

A este respecto, nótese cómo la importancia de reproducir con exactitud el tejido narrativo de L'assaggiatrice e Il conto delle minne ha sido un factor decisivo durante el proceso traductológico. Aun haciéndose traducciones de contenido y de significado con las que se puede estar en mayor o menor desacuerdo, la subjetividad de las ideas de los protagonistas de las historias de Torregrossa ha de ser retratada con cautela si no se quiere desvirtuar el sentido original de las consideraciones textuales. Cualquier mínimo cambio o interpretación errónea podrían acabar desconfigurando la estructura formal del texto.

\section{Conclusiones}

La intención comunicativa del italiano o del siciliano suele coincidir con la del español. Sin embargo, el modo en que estas lenguas construyen sus enunciados puede ser totalmente distinto. Resultado de las rígidas normas gramaticales que codifican el sistema lingüístico de cada idioma, el traductor en ocasiones se ha visto en la necesidad de modificar la estructura del texto de origen en favor del plano semántico -ha renunciado a la microestructura textual en pro de la macroestructura-. La captación del significado del mensaje de Torregrossa constituye, en consecuencia, el elemento basilar sobre el que se han llevado a cabo

Simonetta Agnello Hornby. Basta pensar en La Mennulara para comprobarlo: "[N]on è facile definirla, senza dubbio era dotata di notevole intelligenza nonché di una certa cultura: una donna complessa. A casa si rideva della sua segretezza: mio padre, che era nell'arma dei Carabinieri, sosteneva che se fosse nata maschio sarebbe diventata capomafia, la chiamava 'fimmina di panza'”' (Agnello Hornby 2004: 135). 
las traducciones de L'assaggiatrice y de Il conto delle minne. Solo de esta manera ha sido posible interpretar ambos relatos y traducirlos con cuidado y esmero a los lectores hispanófonos.

Para lograr este objetivo, se ha hecho asimismo indispensable la realización de adaptaciones léxicas o gramaticales que respeten la trama original y la estructura formal de las novelas de Torregrossa, libros en los que el trasfondo femenino y el lenguaje mafioso ocupan un lugar prominente. Prueba de ello han sido los términos attapanata y femmina di panza, dos expresiones que a priori podrían parecer difíciles de entender por parte de un lector hispano (o incluso italiano). Los problemas generados por la traducción al castellano de estos conceptos se han constatado en: 1) el empleo del adjetivo jergal castellano enchironada para aludir al estado de confusión, vergüenza y falta de libertad expresadas por el término siciliano attapanata; y 2) el uso en español de mujer reservada frente al sicilianismo femmina di panza, formas, ambas, ligadas al silencio, a la sumisión y al secretismo insulares. En estos dos casos el siciliano se convierte en un símbolo de la "mafiosidad" y de la opresión femenina isleñas, es decir, en un instrumento comunicativo que, en respuesta a la pregunta que da título a este trabajo, ha puesto de manifiesto los sufrimientos padecidos por muchas sicilianas a lo largo de la historia.

\section{Referencias bibliográficas}

Agnello Hornby, Simonetta (2004). La Mennulara, Milano: Feltrinelli [primera edición 2002].

Bouzalmate, Hussein (1995). Traducción y empatía, en: Encuentros complutenses en torno a la traducción [ed. Rafael Martín Gaitero], Madrid: Editorial Complutense, pp. 93-97.

Di Bella, Maria Pia (2011). Dire o tacere in Sicilia. Viaggio alle radici dell'omertà [pref. di Luigi Maria Lombardi Satriani], Roma: Armando Editore.

Dino, Alessandra (1998). Donne, mafia e processi di comunicazione, en: Rassegna Italiana di Sociologia, XXXIX (n. 4), pp. 477-512.

Dino, Alessandra (2000). Donne di Cosa Nostra, en: Nuove Effemeridi, XIII (n. 50/ II), pp. 74-91.

Dino, Alessandra / Meli, Angelo (1997). Silenzi e parole dall'universo Cosa Nostra. Il ruolo delle donne nella gestione dei processi di comunicazione, Palermo: Sigma Edizioni.

Faccioli, Francesca (1983). L'immagine della donna criminale, en: Dei Delitti e delle Pene, I (n. 1), pp. 110-133.

Fiandaca, Giovanni (2003) (a cura di). Donne e mafia. Il ruolo delle donne nelle organizzazioni criminali, Palermo: Università degli studi di Palermo.

González de Sande, Mercedes (2009). La narrativa de Carmen Martín Gaite en Italia: Nubosidad variable y la problemática y metodología de su traducción al italiano, en: Destiempos, n. 19, pp. 459-487.

Istituto dell'Enciclopedia Italiana Fondata da Giovanni Treccani (2020). Vocabolario 
online, <https://www.treccani.it/vocabolario/> (02/12/2020).

Martín Gaitero, Rafael (1995) (ed.). Encuentros complutenses en torno a la traducción, Madrid: Editorial Complutense.

Mounin, Georges (1999). Teoria e storia della traduzione [trad. di Stefania Morganti], Torino: Einaudi.

Newmark, Peter (1977). Communicative and Semantic Translation, en: Babel. Revue Internationale de la Traduction, n. 23/4, pp. 163-180.

Newmark, Peter (2010). Manual de traducción [vers. española de Virgilio Moya], Madrid: Cátedra [primera edición 1992].

Pasculli, Antonella (2009). Il ruolo della donna nell'organizzazione criminale, en: Rivista di Criminologia, Vittimologia e Sicurezza, III (n. 2), pp. 80-97.

Strazzeri, Irene (2016). Se l'antimafia è donna. Il potere della parola femminile nell'affermazione di una cultura della legalità, en: Nómadas. Revista Crítica de Ciencias Sociales y Jurídicas, Vol. especial: Mediterranean Perspectives (n. 49), pp. 167-174.

Torregrossa, Giuseppina (2010a). L'assaggiatrice, Soveria Mannelli (Catanzaro): Rubbettino [primera edición 2007].

Torregrossa, Giuseppina (2010b). Il conto delle minne, Milano: Mondadori [primera edición 2009].

Tropea, Giovanni (1990) (diretto da). Vocabolario siciliano. Vol. III (N-Q), Palermo: Centro di studi filologici e linguistici siciliani.

Varvaro, Alberto (2014). Vocabolario Storico-Etimologico del Siciliano (VSES) (2 vols.), Strasbourg: Éditions de linguistique et de philologie, Centro di studi filologici e linguistici siciliani.

\section{"Le femmine di panza si sentono attapanate?": Reflections on the translation of the Spanish interpretation of Sicilian literary language}

Giuseppina Torregrossa's literary language distills the defining essence of her homeland: Sicily. The 'Sicilianness' of her texts summons up the oppressive nature of the woman islander and underlines the 'mafiosity' of a territory which occasionally lives anchored in the secrecy of silence. With the focus on these cultural characteristics, this article addresses the feminine spirit of the island, not always easy to disclose to Hispanic readers. From a practical and translational vein, this piece of research emphasizes the difficulties that arise when two typically Sicilian expressions are rendered into Spanish: attapanata ('locked away') and femmina di panza ('reserved woman'). The analysis of these two cases leads us to ancient Sicilian culture and allows us to give an answer to the title of this article: Le femmine di panza si sentono attapanate? 'Do reserved women feel locked away?'

Key words: translation practice, translation quandaries, Sicilian culture, Sicilian-Spanish translation, Giuseppina Torregrossa. 
